\title{
Lithography-free disordered metal-insulator-metal nanoantennas for colorimetric sensing
}

\author{
Zeinab Eftekhari, ${ }^{1,2}$ (1) Amir Ghobadi, ${ }^{1,3}$ (1) and Ekmel Ozbay ${ }^{1,2,3, *}$ \\ ${ }^{1}$ NANOTAM-Nanotechnology Research Center, Bilkent University, 06800 Ankara, Turkey \\ ${ }^{2}$ Physics Department, Bilkent University, 06800 Ankara, Turkey \\ ${ }^{3}$ Department of Electrical and Electronics Engineering, Bilkent University, 06800 Ankara, Turkey \\ *Corresponding author: Ozbay@bilkent.edu.tr
}

Received 15 September 2020; revised 7 November 2020; accepted 10 November 2020; posted 11 November 2020 (Doc. ID 410213 ); published 10 December 2020

\begin{abstract}
The colorimetric detection of bio-agent targets has attracted considerable attention in nanosensor designs. This platform provides an easy to use, real-time, and rapid sensing approach, as the color change can be easily distinguished by the naked eye. In this Letter, we propose a large scale compatible fabrication route to realize colorimetric optical nanosensors with a novel configuration. For this purpose, we design and fabricate a tightly packed disordered arrangement of Fabry-Perot based metal-insulator-metal nanoantennas with a resonance frequency at visible light wavelengths. In this design, the adsorbed bio-agent changes the effective refractive index of the cavity, and this causes a shift in the resonance wavelength. The experimental data show that the proposed design can have sensitivity values $>70 \mathrm{~nm} /$ refractive index unit. Unlike other optical sensing schemes that rely mainly on hot spot formation and field enhancement, this design has a large active area with relatively uniform patterns that make it a promising approach for low-level and reliable bio-detection. () 2020 Optical Society of America
\end{abstract}

https://doi.org/10.1364/OL.410213

Biosensors have revealed their tremendous potential in many applications, including medical diagnosis, food safety, and environmental monitoring. However, their main use is in the detection of analytes and bio-agents such as disease biomarkers and contaminants. Based on the detected signal, biosensors can be classified as optical (such as plasmonic biosensors), electrochemical, piezoelectric, or magnetic biosensors [1,2]. Among all biosensors, optical biosensors are one of the most commonly used tools for analytical biosensing due to their high sensitivity, specificity, potential for real-time sensing, and cost effectiveness $[3,4]$.

One the most commonly used colorimetric optical sensing schemes is based on localized surface plasmon resonances (LSPRs) and propagating surface polaritons (PSPs), since noble metal nanostructures show LSPR in the visible frequencies [5-11]. In this configuration, upon the excitation of LSPRs, absorption and scattering cross sections of noble metallic nanoparticles are greatly enhanced, and extremely intense and localized electromagnetic fields are created in "hot spots" [12-15]. These high intensity spots enable the devices to track any small change in the dielectric environment surrounding these nanostructures [9]. However, this can also be considered as a drawback for these systems. The non-uniform lateral field intensity in these designs causes a limitation on their detection capability $[12,14]$. For example, in a periodic design, we have high field localization in the gap between units and low enhancement at the top of the units. Therefore, depending on the location of a bio-agent with respect to the unit, the field enhancement and resonance shift can change. Although a single plasmonic antenna can also be used for detection, the probability of overlapping of a molecule with this single element is quite low. That is why they are not a viable solution for practical use. Besides this technical limitation, these plasmonic sensors are mainly high-cost precious-metal based designs or their fabrication demands expensive tools such as electron beam lithography (EBL), which is not a large scale compatible tool. It should be noted that some innovative EBL-free designs have been proposed in recent years $[16,17]$. Moreover, dielectric based metasurfaces were found to be promising to replace the precious-metal based plasmonic sensors [18-21].

The above-mentioned scalability requirement can be solved by planar design since they do not need any lithography or patterning. Strong optical interference in metal-insulator (MI) and metal-semiconductor (MS) pair based designs can be used to achieve ultra-broadband perfect absorbers and narrowband color filters $[22,23]$. The most widely used cavity design color filter is a MI-metal (MIM) configuration. In a typical MIM design, a specific wavelength is absorbed by the cavity and the rest of the spectrum gets reflected. This creates a set of subtractive colors called the CMY (cyan-magenta-yellow) model. In a recent paper, we showed that high efficiency RGB (red-greenblue) colors can be made using Bi metamaterials [24]. However, the resonance frequency of an MIM color filter is defined by the spacer layer thickness and refractive index, which has no contact with the surrounding environment. That is why these 
planar cavity designs have been rarely used for sensing applications [25-27]. To be able to use them in sensing applications, nanostructuring is needed to provide an area for interaction with the surrounding environment. However, in the meantime, the fabrication route for nanostructuring should be large scale compatible.

In this Letter, we develop a lithography-free nanostructured MIM design for colorimetric sensing application. In other words, the proposed design is a Fabry-Perot (FP) based disordered arrangement of densely packed MIM nanoantennas. In this design, the target analyte can diffuse inside the gaps and tune the spacer effective refractive index and this, in turn, causes a peak resonance shift and color change. To design and analyze this system, we first perform finite-difference-timedomain (FDTD) simulations on a planar configuration. Later, to estimate the effect of the induced randomness, a transfer matrix method (TMM) theoretical analysis is conducted. Finally, the optimized design is fabricated and characterized. To obtain a nanostructured spacer layer, the oblique angle deposition technique is used, which is a room temperature and large scale compatible approach with no material limitation. Based on the obtained optical results, visible light colors can be realized with this MIM antenna design. Upon deposition of a dielectric layer (as the modeling agent for biomarkers), the resonance frequency is red-shifted, and a sensitivity as high as $\sim 84 \mathrm{~nm} /$ refractive index unit (RIU) can be achieved. Moreover, the color (position of resonance peak) of the MIM biosensor can be passively adopted by the spacer layer thickness. The proposed design strategy offers a highly sensitive, robust, and compact biomolecular diagnostics with a real-time, label-free, and early point-of-care diagnosis that can be used in various applications such as drug detection and environmental monitoring.

To gain insight on the resonance condition of a Bi based MIM design, we initially begin our analysis using a planar design model, as shown in Fig. 1(a). The spacer layer is made of lithium fluoride $(\mathrm{LiF})$ with a thickness of $D_{I}$. The bottom and top layers are $\mathrm{Bi}$. The top layer thickness is $D_{M}$, while the bottom layer is an optically thick layer with no transmission in the visible range. To realize the influence of the geometrical dimensions upon the absorption response, we utilized the Lumerical (FDTD) simulation package. In the FDTD simulations, we illuminated the unit cell with a broadband plane wave at normal incidence whose electric field is polarized in the $x$ direction. Prior to these simulations, the permittivity values of bulk $\mathrm{Bi}$ and $\mathrm{LiF}$ layers were experimentally extracted using a spectroscopic ellipsometer, as explained in detail in our previous work [24]. The permittivity data of Bi are shown in Fig. 1(b). Due to superior matching of the Bi permittivity data with that of an ideal absorber, the MIM design can absorb all of the frequencies except for the ones with constructive interference. This can be seen from the absorption contour plot shown in Fig. 1(c). As this panel implies, the incident light is dominantly absorbed in the top layer, and it shows a notch in the middle of the simulated spectrum. With this design, a spectrally selective reflection peak can be achieved, and a reflective RGB color filter can be realized. To optimize the color purity, we need to reduce the full-width-at-half-maximum (FWHM) of the reflection peak and broaden the absorption bands to avoid multi-color reflection. For this purpose, the top layer thickness is initially swept while the spacer layer thickness is fixed at $150 \mathrm{~nm}$. Figure $1(\mathrm{~d})$ shows the dependence of the reflection spectra of the MIM design to $D_{M}$. As implied from
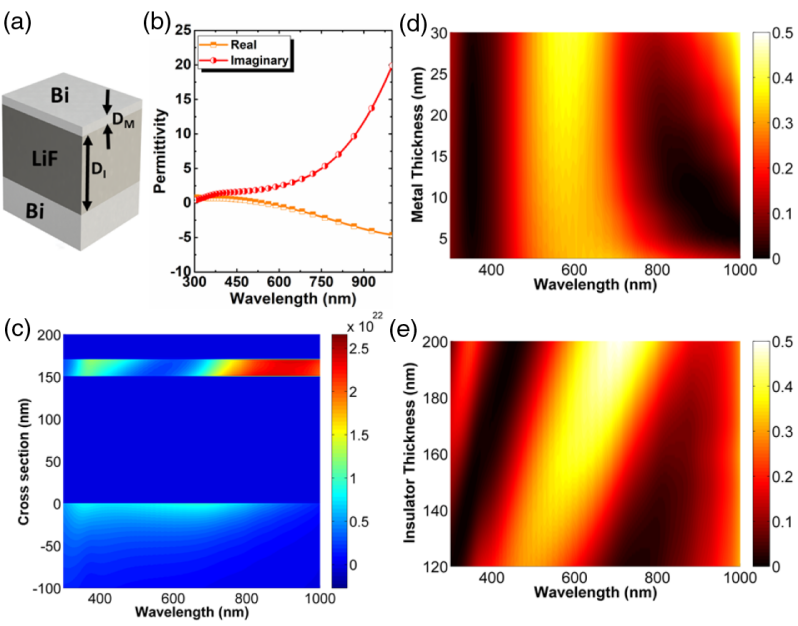

Fig. 1. (a) Schematic representation of the proposed MIM cavity with its (b) extracted spectral permittivity of $\mathrm{Bi}$ (both real and imaginary) in our desired range of 300-1000 nm. (c) Two-dimensional profile of the absorbed power across the MIM cavity as a function of incident light wavelength. Sweep and optimization of reflection spectra for different geometries of (d) $D_{M}$ (while $D_{I}$ is fixed at $150 \mathrm{~nm}$ ) and (e) $D_{I}$ (while $D_{M}$ is fixed at $20 \mathrm{~nm}$ ).

this plot, as $D_{M}$ increases, the FWHM of reflection gradually narrows. However, at large dimensions, a new reflection peak starts to appear in off-resonance frequencies. Therefore, a tradeoff should be followed to have the best color purity, and for this, $D_{M}$ is depicted as $20 \mathrm{~nm}$. In the next step, the spacer layer influence on the reflection response of the MIM cavity is investigated, as shown in Fig. 1(e). In this panel, the reflection peak experiences a nearly linear red shift as the thickness increases. Therefore, based on the desired color, the $D_{I}$ can be modified to tune the spectral position of the reflection peak.

All of the above-mentioned results prove the RGB color generation of a planar Bi based MIM cavity. Yet, the spectral position of this MIM cavity is defined by the spacer optical thickness (both thickness and refractive index). Therefore, a change in color requires a tuning on the spacer layer. However, in a planar configuration, this layer is capped by the top metal absorber, and it has no interaction with an outside dielectric region that is air. Due to this reason, it cannot be a good option for the colorimetric optical sensing of an unknown agent. To make it functional for sensing purposes, nanostructuring should be introduced into this architecture to enhance its active surface area. In other words, the planar MIM layer should be split into sub-wavelength MIM nanoantennas with ultra-small gaps for sensing agent diffusion.

Therefore, instead of a bulk LiF layer, we need to have nanorod (NR) designs. However, the question is, "Can we keep the reflective color generation property of planar MIM design in a nanoantenna MIM configuration?" Based on effective medium theory, sub-wavelength dielectric geometries can be modeled as a bulk system with an effective refractive index that is a value between air and dielectric indices, as shown in Fig. 1(a). To predict the absorption response of this effective medium MIM design, we use a modeling approach based on TMM. As explained in detail in our previous studies [22,24] in this model, we fix the geometries $\left(D_{I}=150 \mathrm{~nm}\right.$ and $\left.D_{M}=20 \mathrm{~nm}\right)$ and the spacer layer material (as LiF) and look for an ideal absorber material data (with near zero reflection) for the top 
coating. Then, we compare this ideal material permittivity data with that of Bi. Figure 1(b) shows our model used in TMM modeling. For this design, the reflection contour plots have been extracted as a function of top material real $[\operatorname{Re}(\varepsilon)]$ and imaginary $[\operatorname{Im}(\varepsilon)]$ permittivity parts. In these panels, for instance, staying within a 0.1 circle means that the MIM design is below $10 \%$ reflection at that wavelength. These contour plots have been acquired for three different spacer effective refractive indices $\left(N_{\text {effective }}\right)$ of 1.4, 1.3, and 1.2 to simulate different LiF NR filling fractions. The resonance wavelengths of 350 and $800 \mathrm{~nm}$ are chosen for these plots, since the light absorption of the $\mathrm{Bi}$ top layer is maximum at these wavelengths. For $350 \mathrm{~nm}$ incident light wavelength [Fig. 2(c)], reducing the $N_{\text {effective }}$ causes a slight shift in the reflection circles. However, in all three conditions, Bi data stay within a 0.2 circle. The same holds for the $800 \mathrm{~nm}$ excitation, as exhibited in Fig. 2(d). Therefore, nanostructuring of LiF does not ruin the absorption optical response of the MIM cavity. Specifically, in the case of high $N_{\text {effective }}$, we can have a quite similar response with the planar design. This implies that higher packing density is more favorable. On the other hand, in all panels, the ideal point (zero reflection point) shifts toward more negative $\operatorname{Re}(\varepsilon)$, as the effective refractive index increases. Looking at the Bi permittivity data, we can see that as the wavelength increases, the $\operatorname{Re}(\varepsilon)$ moves toward negative values. This matching is quite promising from sensing the point of value. This means that as we expose these MIM nanoantennas to a biomarker adsorption, the $N_{\text {effective }}$ increases and the reflection dip shifts toward longer wavelengths. All of these theoretical calculations predict the high functionality of a tightly packed MIM nanoantenna absorber in colorimetric sensing.

In the next step, we have to find a fabrication route to realize these LiF NRs in large scales. Among the various large scale compatible nanostructuring techniques, oblique angle deposition is

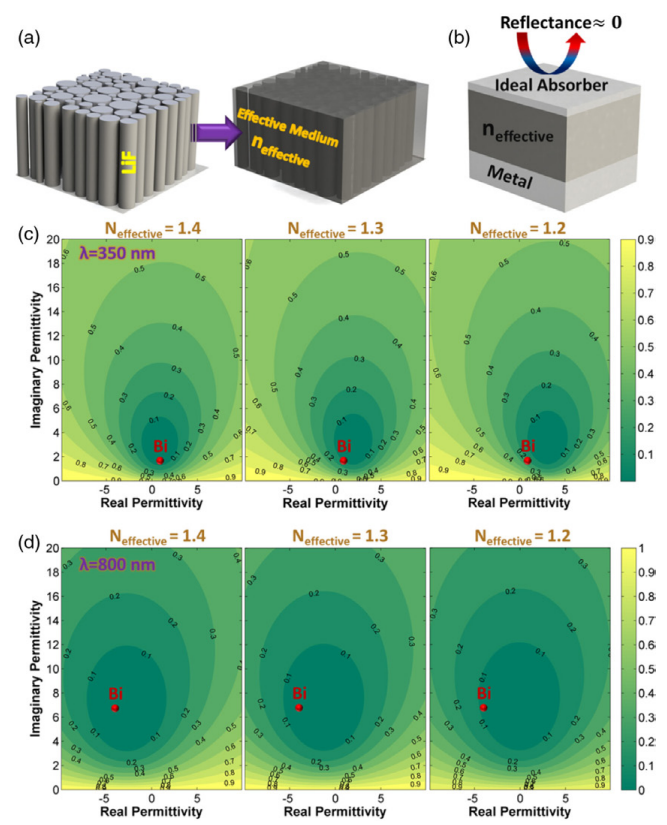

Fig. 2. (a) Schematic illustration of the real LiF NR design and its effective medium model. (b) MIM ideal absorber used to extract the reflection contour plots. TMM extracted reflection contour plots for three different $N_{\text {effective }}$ of 1.4, 1.3, and 1.2 for incident light wavelengths of (c) $350 \mathrm{~nm}$ and (d) $800 \mathrm{~nm}$. an effective approach to fabricate nanostructures with no material restriction.

Specifically, for dense high aspect ratio structures, where the EBL becomes challenging, this approach can provide a robust solution. Figure 3(a) schematically depicts the proposed MIM nanoantenna design with multiple NR radii. Every NR is capped with a thin $\mathrm{Bi}$ layer. To realize this design configuration, we first deposit a $150 \mathrm{~nm}$ Bi layer on a diced Si substrate. This layer is coated by the thermal evaporation technique (VAKSIS tool). The deposition rate is set at $\sim 2 \AA / s$, and the chamber pressure is kept below $5 \mathrm{E}-6$ Torr throughout the process. Afterward, oblique angle deposition is utilized to fabricate LiF NRs, as explained in detail in our previous study [24]. The deposition conditions are the same as the $\mathrm{Bi}$ layer, except that the rate is $5-6 \AA / s$ to create a better nanotexturing. Finally, a $20 \mathrm{~nm}$ thick Bi layer is coated on top of the NRs to complete the fabrication of disordered MIM nanoantennas. It should be noted that the size of the samples are $1 \mathrm{~cm} \times 1 \mathrm{~cm}$. Due to the large scale compatibility of the fabrication route, this dimension can be up scaled. To verify the formation of this hybrid design, scanning electron microscopy (SEM) imaging is utilized. Figure 3(b) exhibits the existence of standing tightly packed LiF NRs with sub-wavelength radii. According to Fig. 3(c), the deposition of a Bi thin film has not led to continuous layer formation, and the surface morphology has not changed. Therefore, a biomarker analyte can diffuse within its small gaps and tune the cavity's effective index. In this way, upon adsorbent deposition, the resonance wavelength shifts and consequently the color changes. This is graphically explained in Fig. 3(d). To demonstrate the biosensing capability of our disordered MIM nanoantenna, a model design is used, as shown in Fig. 3(e). To model these bioagent analytes, we spin-coated the MIM cavity with 495Poly (methyl methacrylate) (495PMMA) A2 to coat the NR surfaces and the gaps in between, as depicted in the SEM image in Fig. 3(f).

To evaluate the colorimetric response of this disordered MIM nanoantenna array design, the optical reflection response of the structure is evaluated for three different $D_{I}$ values of 140,170 , and $200 \mathrm{~nm}$ [Figs. 3(g)-3(i)]. The three different thicknesses are used to generate three different colors. The reflection spectra of these MIM nanoantennas, in the range of 350-950 nm, before and after PMMA coating are measured using a homemade setup. In this setup, a halogen lamp is used as the light source, integrated with a microscope to collect the reflection data. These collected reflection data are transferred into a spectrometer (Newport Corporation; Irvine, CA, USA) (Newport OSM2). In all of the measurements, the obtained reflection values are normalized with that of a thick $\mathrm{Al}$ sheet. To have a better visualization, the optical image of the samples before and after coating are shown on top of the spectral reflection plot. For the case of $D_{I}=140 \mathrm{~nm}$, the PMMA coating has caused a $39 \mathrm{~nm}$ shift in the reflection peak position. Taking the PMMA refractive index to be $\sim 1.5$ in these frequencies, the maximum experimental sensitivity of the nanosensor is recorded as $S_{\max }=\Delta \lambda / \Delta n=39 \mathrm{~nm} /(1.5-1.0)=72 \mathrm{~nm} /$ RIU. The same characterization is also conducted on $D_{I}=170 \mathrm{~nm}$, and $200 \mathrm{~nm}$, where the resonance red shift is found to be 34 and $42 \mathrm{~nm}$ (corresponding to the $S_{\max }$ values of 68 and $84 \mathrm{~nm} / \mathrm{RIU}$ ).

Overall, this work demonstrates a design strategy to realize a sensitive, real-time, and label-free optical biosensing scheme. The proposed route is a large scale and extendable approach that can be adopted for other MI combinations. The detailed 
(a)
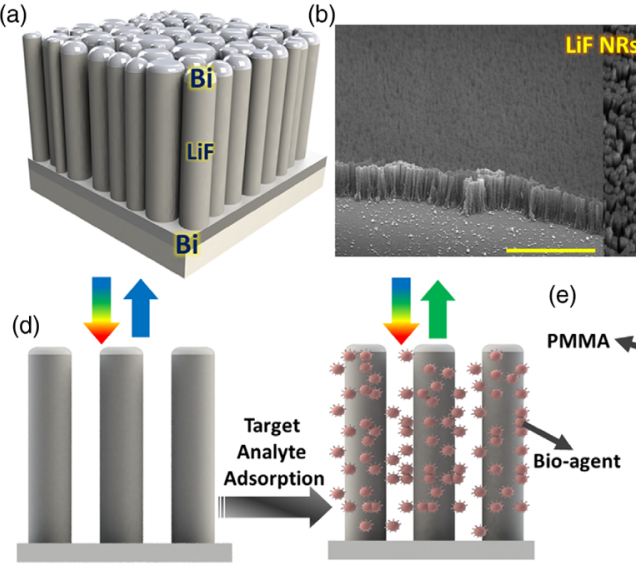

(b)

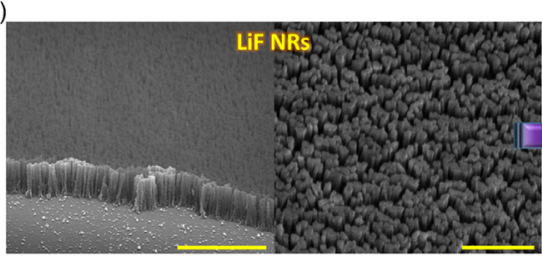

(c)
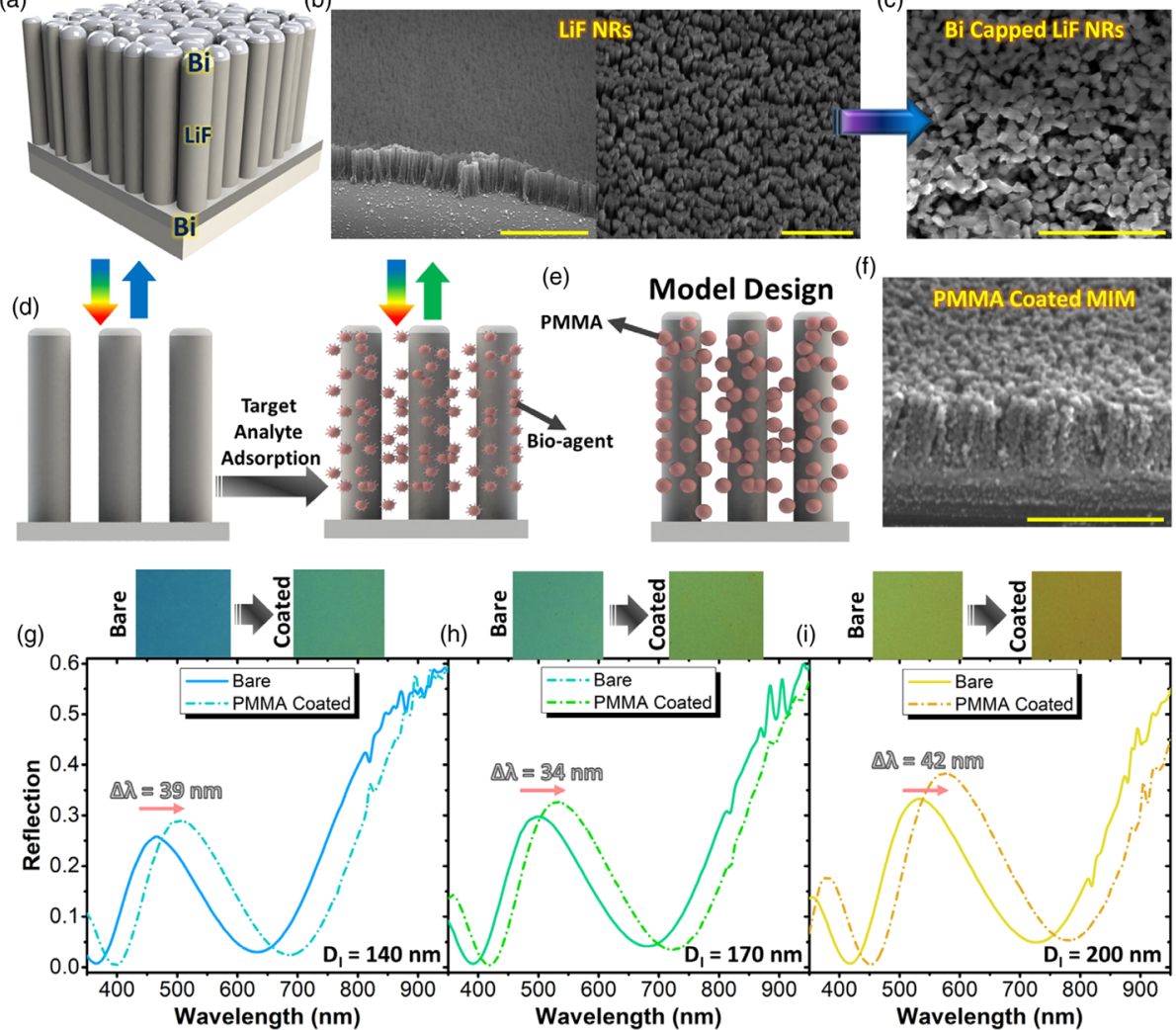

Fig. 3. (a) Simplified schematic illustration of the disordered MIM nanoantennas design. SEM images of (b) bare LiF NRs and (c) those coated with a thin Bi layer. (d) Operation principle of our proposed biosensor. (e) Model design and (f) its cross sectional SEM image. In all of the SEM images, the bar length is $400 \mathrm{~nm}$. Reflection spectra of bare and PMMA coated MIM nanoantenna samples for three different $D_{I}$ values of (g) $140 \mathrm{~nm}$, (h) $170 \mathrm{~nm}$, and (i) $200 \mathrm{~nm}$. At the top of this linear plot, the optical images of bare and PMMA coated samples are depicted.

design, analysis, and characterization results show the unprecedented potential of this random plasmonic platform for many optoelectronic applications.

Disclosures. The authors declare no conflict of interest.

\section{REFERENCES}

1. C. I. L. Justino, A. C. Duarte, and T. A. P. Rocha-Santos, Sensors 17, 2918 (2017).

2. R. Samson, G. R. Navale, and M. S. Dharne, 3 Biotech 10, 385 (2020).

3. J. Liu, M. Jalali, S. Mahshid, and S. Wachsmann-Hogiu, Analyst 145 364 (2020).

4. C. Chen and J. Wang, Analyst 145, 1605 (2020).

5. J. Homola, Chem. Rev. 108, 462 (2008).

6. L. Chen, L. Chan, X. Fu, and W. Lu, ACS Appl. Mater. Interfaces 5, 284 (2013).

7. K. Saha, S. S. Agasti, C. Kim, X. Li, and V. M. Rotello, Chem. Rev. 112, 2739 (2012).

8. P. D. Howes, S. Rana, and M. M. Stevens, Chem. Soc. Rev. 43, 3835 (2014).

9. L. Tang and J. Li, ACS Sens. 2, 857 (2017).

10. G. Qiu, Z. Gai, Y. Tao, J. Schmitt, G. A. Kullak-Ublick, and J. Wang, ACS Nano 14, 5268 (2020).

11. X. Han, K. Liu, and C. Sun, Materials (Basel) 12, 1411 (2019).

12. J. Jiang, X. Wang, S. Li, F. Ding, N. Li, S. Meng, R. Li, J. Qi, Q. Liu, and G. L. Liu, Nanophotonics 7, 1517 (2018).

13. N. Kongsuwan, X. Xiong, P. Bai, J. Bin You, C. E. Png, L. Wu, and O. Hess, Nano Lett. 19, 5853 (2019).

14. D. Radziuk and H. Moehwald, Phys. Chem. Chem. Phys. 17, 21072 (2015).
15. H. Wei, S. M. Hossein Abtahi, and P. J. Vikesland, Environ. Sci. Nano 2, 120 (2015).

16. C. Q. Nguyen, W. J. Thrift, A. Bhattacharjee, S. Ranjbar, T. Gallagher, M. Darvishzadeh-Varcheie, R. N. Sanderson, F. Capolino, K. Whiteson, P. Baldi, A. I. Hochbaum, and R. Ragan, ACS Appl. Mater. Interfaces 10, 12364 (2018).

17. W. J. Thrift, C. Q. Nguyen, M. Darvishzadeh-Varcheie, S. Zare, N. Sharac, R. N. Sanderson, T. J. Dupper, A. I. Hochbaum, F. Capolino, M. J. Abdolhosseini Qomi, and R. Ragan, ACS Nano 11, 11317 (2017).

18. D. U. Yildirim, A. Ghobadi, M. C. Soydan, M. Gokbayrak, A. Toprak, B. Butun, and E. Ozbay, J. Phys. Chem. C 123, 19125 (2019).

19. O. Yavas, M. Svedendahl, P. Dobosz, V. Sanz, and R. Quidant, Nano Lett. 17, 4421 (2017).

20. N. Bontempi, K. E. Chong, H. W. Orton, I. Staude, D. Y. Choi, I. Alessandri, Y. S. Kivshar, and D. N. Neshev, Nanoscale 9, 4972 (2017).

21. F. Yesilkoy, E. R. Arvelo, Y. Jahani, M. Liu, A. Tittl, V. Cevher, Y. Kivshar, and H. Altug, Nat. Photonics 13, 390 (2019).

22. A. Ghobadi, H. Hajian, B. Butun, and E. Ozbay, ACS Photon. 5, 4203 (2018).

23. C. Ji, K. T. Lee, T. Xu, J. Zhou, H. J. Park, and L. J. Guo, Adv. Opt. Mater. 5, 1700368 (2017).

24. A. Ghobadi, H. Hajian, M. Gokbayrak, B. Butun, and E. Ozbay, Nanophotonics 8, 823 (2019).

25. S. Ayas, G. Bakan, E. Ozgur, K. Celebi, G. Torunoglu, and A. Dana, Opt. Lett. 43, 1379 (2018).

26. M. Serhatlioglu, S. Ayas, N. Biyikli, A. Dana, and M. E. Solmaz, Opt. Lett. 41, 1724 (2016).

27. M. Elkabbash, K. V. Sreekanth, Y. Alapan, M. Kim, J. Cole, A. Fraiwan, T. Letsou, Y. Li, C. Guo, R. M. Sankaran, U. A. Gurkan, M. Hinczewski, and G. Strangi, ACS Photon. 6, 1889 (2019). 